\title{
Is There a Role for an Ultrasonic Bone-Cutting Device in Adult Spinal Deformity: A Safety and Reproducibility Study
}

\author{
Pooria Hosseini, ${ }^{1}$ Gregory M Mundis Jr, ${ }^{1,}$ Robert Eastlack, ${ }^{2}$ Jeff Pawelek, ${ }^{1}$ Stacie Nguyen, ${ }^{1}$ and Behrooz \\ A. Akbarnia ${ }^{1}$ \\ ${ }^{1}$ Orthopedic Department, San Diego Center for Spinal Disorders, San Diego, USA \\ ${ }^{2}$ Orthopedic Department, Scripps Health, CA, USA \\ "Corresponding author: Gregory M Mundis Jr, Orthopedic Department, San Diego Center for Spinal Disorders, San Diego, USA. Tel: +858-5547988, Fax: +858-5548231, E-mail: \\ gmundis1@gmail.com
}

Received 2016 June 06; Accepted 2016 July 10.

\begin{abstract}
Background: Ultrasonic bone-cutting devices (UBC) are new cutting tools and have low frequency ultrasonic blade. There is limited data on the safety and effectiveness of using ultrasonic bone-cutting devices in the treatment of adult spinal deformities (ASD).

Objectives: This Retrospective review of prospectively collected data was designed to determine if the use of an ultrasonic bonecutting device is safe in the adult spinal deformity population and to compare its effectiveness in blood loss reduction by using a comparison group from a prospective multicenter database of adult spinal deformity patients.

Methods: Nineteen consecutive surgical ASD cases in which the UBC was used were compared with 19 propensity-matched cases from a prospective ASD database in which conventional bone cutting instruments were used. The two groups were matched based on age, ASA, and number of levels fused posteriorly. The need for blood transfusion, volume of blood transfusion if required, estimated blood loss (EBL), and total operating time were compared between the two groups. Data were analyzed using non-parametric Mann-Whitney U test and Spearman's Correlation test $(\mathrm{P}<0.05)$.

Results: There was no statistically significant difference in any measured parameter between the two groups. While the EBL difference between the two groups ( $925 \mathrm{~mL}$ in the study group vs. $1628 \mathrm{~mL}$ in the control group) was not statistically significant $(\mathrm{P}=0.142)$, the $703 \mathrm{~mL}$ difference is clinically relevant. In addition, no complications directly related to the use of the UBC were reported.

Conclusions: The use of an ultrasonic bone-cutting device was shown to be safe and effective in the surgical treatment of ASD. It resulted in a $43 \%$ reduction in EBL, which was clinically relevant and statistically non-significant, without the addition of any complications. We did not identify statistical differences in transfusion rates, EBL, or operative time, which may be due to our small sample size.
\end{abstract}

Keywords: Adult Spine Deformity, Ultrasonic Bone-Cutting Device, Bone Scalpel, Blood Loss

\section{Background}

The safety and reproducibility of performing osteotomies is important in achieving adequate spinal decompression and deformity correction. However, due to the high technical nature of osteotomies such as facetectomy, Smith-Petersen osteotomy, and pedicle subtraction osteotomy, the use of traditional surgical tools including high-speed drills, rotating burs, and thread-wire saws, carry a risk of associated intraoperative complications such as durotomy, spinal cord or nerve root injury, visceral and vascular injury, and excessive blood loss (1-3). There is also an inherent inefficiency with the use of these surgical instruments (4).

Ultrasonic devices were originally developed for dentistry in 1952. They were very quickly adopted, and their use expanded. By the 1970s the technology allowed for effective debulking and removal of soft tissue tumors (5,
6). These new cutting tools are low frequency ultrasonic blades. They cut through dense and mineralized tissues and spare more compliant adjacent soft tissues. The device is comprised of a blunt ultrasonic blade with self-irrigation system that oscillates at above 22,500 cycles/ $s$ (7).

There is limited data about its application in spine surgery, and no published literature data for its use in the surgical correction of adult spinal deformity. The main advantages reported include reduction of blood loss and operating time $(1,8-10)$. However, other authors have reported an increase in operating time $(9,11)$. The lack of a control group in the existing literature obviates the need for a case-matched comparative study $(1,10)$. To the best of our knowledge the only study published where a control group was used was by Bartley et al. (12). In this study, single surgeon cases, including 60 adolescent idiopathic scoliosis (AIS) treated with posterior spinal fusion, 
divided into three groups of 20 ultrasonic bone-cutting device (UBC) group, 20 Cobb matched cases, and 20 most recent cases prior to surgeons using UBC were compared. They concluded that the use of UBC results in significantly less bleeding compared with the control group and limits the overall blood loss by $30 \%-40 \%$.

\section{Objectives}

The purpose of this study is to determine if the use of UBC is safe in the adult spinal deformity population and to compare its effectiveness in blood loss reduction using a propensity-matched control group.

\section{Methods}

\subsection{Patients}

This study was reviewed and approved by an institutional review board prior to initiation. Data was retrospectively collected from medical records of 19 consecutively operated patients from September 2013 to March 2014 in a single center, in which the UBC (Misonix, Inc., NY, USA) was used to perform any type of osteotomy during the operation. The majority of patients were diagnosed with kyphoscoliosis. UBC was used twice in one of the cases due to the staged nature of the operation. All UBC cases were propensity matched to a group of patients where traditional instruments were used. The control group was selected from a consecutively enrolled prospective adult spinal deformities (ASD) database. The control group surgeries were performed between October 2009 and October 2012. The control group included osteotomies performed with high-speed drills, rotating burs, and thread-wire saws. The control group was propensity matched based on age, ASA, and number of levels fused posteriorly. The need for blood transfusion, volume of blood transfusion if required, estimated blood loss, and total operating time parameters was collected.

In addition, the UBC related complications data including incidental durotomy, neuromonitoring changes, and unintended harm to adjacent tissues were collected.

\subsection{Surgical Technique}

Intraoperatively, once the subperiosteal dissection was complete and the bony surfaces were cleared of soft tissue, the UBC was used at all levels to perform the inferior facetectomy followed by removing the cartilage from the superior articulating process (SAP). The interspinous ligaments were removed at all osteotomy sites, followed by resection of the ligamentum flavum. Next, the UBC was used to complete the osteotomy by cutting across the SAP, completing the posterior release. In scoliosis cases the bony remainder of the SAP was left in place as a source of bone graft, and in kyphosis cases the SAP was removed to allow for reduction of the kyphosis.

\subsection{Statistical Analysis}

Mann-Whitney U test was used to test for significant differences between the two groups among continuous parameters. The Spearman rank test was used for categorical parameters. P value less than 0.05 was considered as statistically significant. Non-parametric tests were more appropriate in this study due to small sample size, resulting in non-normal distribution of data.

\section{Results}

A total of 19 consecutive patients ( 14 female and 5 male) were included for analysis in the study group. The mean age at surgery was 61 years (range, 28 - 79 years). They were matched with 19 patients ( 15 female, 4 male) with a mean age of 55 years (range, 19 - 77 years; $P=0.271$ ). Average number of fusion levels in UBD was 9.6 vs. 8.8 in the control group ( $\mathrm{P}=0.583)$; ASA grade was 2.5 and 2.4 , respectively $(\mathrm{P}=0.840)$ (Tables 1,2 and 3$)$.

Average EBL in the study group was $925 \mathrm{~mL}$ vs. $1628 \mathrm{~mL}$ (43.1\% reduction in $\mathrm{EBL})$ in the control group $(\mathrm{P}=0.142)$ and the average total operating time was only 2 minutes less in bone scalpel group compared with control group (425 minutes in bone scalpel group vs. 427 minutes in control group with p value of 0.488 ). Fourteen of the 19 patients in each group required a blood transfusion post operation ( $P$ $=1.00$ ). The average transfusion volume in the bone scalpel group was $1330 \mathrm{ml}$ vs. $1447 \mathrm{ml}$ in control group $(\mathrm{P}=0.571)$. However, clinically it was very noticeable that the bleeding from the bony surfaces was significantly less compared to traditional techniques of performing this same procedure. Figure $1 \mathrm{~A}-1 \mathrm{~B})$. There were no complications associated with the use of the UBD (e.g. incidental durotomy, neuromonitoring changes, unintended harm to adjacent tissues, etc.)

\section{Discussion}

Adult spinal deformity surgery often requires resection of large amounts of bone through various osteotomy techniques, and these maneuvers have traditionally been associated with increased morbidity of the operative procedure. Spinal osteotomies have been associated with greater blood loss, durotomies, and neural deficits $(2,3,13)$. Several different tools are used to perform osteotomies in 
Table 1. Summary of Findings

\begin{tabular}{|c|c|c|c|c|}
\hline Findings & UBC $^{\text {a }}$ Group & Control Group & $\Delta$ & PValue $^{\text {b }}$ \\
\hline Mean Age, $y$ & 61 & 55 & 6 & 0.271 \\
\hline No. of levels fused & 9.6 & 8.8 & 0.8 level & 0.583 \\
\hline ASA & 2.5 & 2.4 & 0.1 & 0.840 \\
\hline $\mathrm{EBL}^{\mathrm{c}}, \mathbf{m L}$ & 925 & 1628 & 703 & 0.142 \\
\hline Operation time, minute & 425 & 427 & 2 & 0.488 \\
\hline Transfusion need & $14 / 19$ & $14 / 19$ & 0 & 1.00 \\
\hline Transfusion volume & 1330 & 1447 & 117 & 0.571 \\
\hline
\end{tabular}

${ }^{\mathrm{a}}$ Ultrasonic bone-cutting device.

${ }^{\mathrm{b}} \mathrm{P}<0.05$ to be statistically significant.

${ }^{\mathrm{c}}$ Estimated blood loss.

Table 2. Bone-Cutting Device Group

\begin{tabular}{|c|c|c|c|c|c|c|c|c|c|c|c|}
\hline Patient & Age, $y$ & Gender & Diagnosis & \# of Levels Fused & PSFL $^{\mathbf{a}}$ & $\operatorname{SPO}(\#)^{\mathbf{b}}$ & ${ }_{3} \operatorname{co}^{\mathrm{C}}$ (yes/no) & $\mathrm{EBL}^{\mathrm{d}}, \mathrm{mL}$ & ASA & OR Time, Min & Transfusion Volume, $\mathbf{m L}$ \\
\hline 1 & 71 & $\mathrm{~F}$ & Kyphoscoliosis & 4 & L1-L5 & 0 & No & 100 & 1 & 301 & 0 \\
\hline 2 & 28 & $\mathrm{~F}$ & Kyphoscoliosis & 6 & T1-T7 & 4 & No & 200 & 1 & 328 & 0 \\
\hline 3 & 79 & $\mathrm{~F}$ & Kyphoscoliosis & 14 & T4-S1 & 4 & No & 2500 & 3 & 730 & 2800 \\
\hline 4 & 60 & $\mathrm{~F}$ & Kyphoscoliosis & 7 & T11-S1 & 0 & Yes & 1300 & 3 & 520 & 700 \\
\hline 5 & 76 & $\mathrm{~F}$ & Kyphoscoliosis & 7 & T11-S1 & 2 & No & 1450 & 2 & 568 & 1050 \\
\hline 6 & 67 & $\mathrm{~F}$ & Kyphoscoliosis & 14 & T4-S1 & 7 & No & 400 & 3 & 358 & 1750 \\
\hline 7 & 32 & $\mathrm{~F}$ & Neuromuscular Scoliosis & 16 & T2-S1 & 8 & No & 800 & 3 & 506 & 700 \\
\hline 8 & 41 & $\mathrm{~F}$ & Adult idiopathic scoliosis & 12 & T2-L2 & 7 & No & 600 & 1 & 365 & 700 \\
\hline 9 & 34 & M & Adult idiopathic scoliosis & 11 & T2-L1 & 0 & No & 1500 & 2 & 437 & 0 \\
\hline 10 & 75 & M & Kyphoscoliosis & 3 & T9-T12 & 1 & No & 200 & 2 & 248 & 0 \\
\hline 11 & 79 & $\mathrm{~F}$ & Cervicothoracic Kyphosis & 12 & $\mathrm{C} 2-\mathrm{T} 7$ & 4 & No & 500 & 3 & 529 & 350 \\
\hline 12 & 65 & M & Kyphoscoliosis & 7 & T11-S1 & 2 & No & 1000 & 2 & 255 & 2450 \\
\hline 13 & 65 & $\mathrm{~F}$ & L3 pathological fracture Multiple Myeloma & 5 & L1-S1 & 0 & No & 700 & 4 & 316 & 1711 \\
\hline 14 & 62 & M & Kyphoscoliosis & 14 & T4-S1 & 0 & Yes & 1500 & 3 & 695 & 2612 \\
\hline 15 & 74 & M & Degenerative disc disease & 9 & T4-L1 & 1 & No & 500 & 4 & 301 & 0 \\
\hline 16 & 49 & $\mathrm{~F}$ & Kyphoscoliosis & 4 & L2-S1 & 1 & No & 950 & 2 & 660 & 700 \\
\hline 17 & 69 & F & Kyphoscoliosis & 14 & T4-S1 & 3 & No & 1300 & 3 & 518 & 1350 \\
\hline 18 & 73 & $\mathrm{~F}$ & Post traumatic kyphosis & 10 & T8-S1 & 2 & Yes & 1200 & 3 & 610 & 350 \\
\hline 19 & 62 & $\mathrm{~F}$ & Kyphoscoliosis & 14 & T4-S1 & 0 & No & 1400 & 2 & 596 & 1400 \\
\hline
\end{tabular}

adult spinal deformity surgery. The safety of using an ultrasonic bone scalpel (BS) has not been investigated in adult spinal deformity surgery. Our study suggests that the BS is equivalent to traditional osteotomy techniques without the incidence of additional complications.

Precise bony cuts play a critical role in successful decompression and osteotomies in ASD surgeries. Conventional osteotomy tools including high-speed burs, and thread-wire saws and the use of osteotomes and a mallet in the presence midline bony defects rendering the spinal canal/cord vulnerable. Similarly, simply the vibration from the mallet striking the osteotome could pose unnecessary risk of incidental durotomies and /or worse injury to the midline neural elements and spinal cord $(2,3,13)$.

To address these issues the bone scalpel was developed. An ultrasonic aspirator was first used in 1947 for the removal of dental plaques (14) and developed more for dentistry in 1952. In 1978 the ultrasonic bone scalpel was used for the first time in neurosurgery $(5,6)$. Existing ultrasonic bone aspirators like Sonopet have been proven to be safe in bone removal both in spine and intracranial surgeries $(5,15)$. Although Sonopet has relatively narrow tips (1.9 - 2.8 


\begin{tabular}{|c|c|c|c|c|c|c|c|c|c|c|c|}
\hline Patient & Age, $y$ & Gender & Diagnosis & \# of Levels Fused & PSFL $^{\mathbf{a}}$ & $\operatorname{SPO}(\#)^{\mathbf{b}}$ & ${ }_{3} \mathrm{Co}^{\mathrm{c}}$ (yes/no) & $\mathrm{EBL}^{\mathrm{d}}, \mathrm{mL}$ & ASA & OR Time, Min & Transfusion Volume, $\mathrm{mL}$ \\
\hline 2 & 25 & F & Kyphoscoliosis & 10 & T2-T12 & 8 & No & 350 & 2 & 229 & 0 \\
\hline 3 & 19 & F & Kyphoscoliosis & 10 & T2-T12 & 8 & No & 200 & 2 & 187 & 0 \\
\hline 4 & 42 & F & Kyphoscoliosis & 11 & T3-L2 & 5 & No & 550 & 2 & 385 & 0 \\
\hline 5 & 43 & F & Кyphoscoliosis & 5 & T11-L4 & 4 & No & 1000 & 1 & 180 & 0 \\
\hline 6 & 77 & M & Kyphoscoliosis & 7 & T11-S1 & 3 & No & 2500 & 3 & 420 & 1800 \\
\hline 7 & 60 & M & Kyphoscoliosis & 9 & T9-S1 & 4 & No & 3600 & 3 & 626 & 2050 \\
\hline 8 & 35 & $\mathrm{~F}$ & Kyphoscoliosis & 10 & T3-L1 & 5 & No & 600 & 2 & 299 & 950 \\
\hline 9 & 50 & F & Kyphoscoliosis & 8 & T10-S1 & 4 & No & 1150 & 2 & 593 & 669 \\
\hline 10 & 68 & F & Kyphoscoliosis & 8 & T10-S1 & 3 & No & 3650 & 3 & 479 & 1750 \\
\hline 11 & 64 & F & Kyphoscoliosis & 9 & T9-S1 & 5 & No & 550 & 3 & 401 & 1050 \\
\hline 12 & 73 & F & Kyphoscoliosis & 8 & T10-S1 & 4 & No & 600 & 3 & 452 & 1400 \\
\hline 13 & 54 & F & Kyphoscoliosis & 8 & T10-S1 & 5 & No & 1850 & 2 & 650 & 1262 \\
\hline 14 & 63 & F & Kyphoscoliosis & 13 & T5-S1 & 3 & No & 250 & 3 & 779 & 700 \\
\hline 15 & 65 & M & Kyphoscoliosis & 8 & T10-S1 & 4 & No & 3750 & 4 & 732 & 324 \\
\hline 16 & 73 & F & Kyphoscoliosis & 8 & T10-S1 & 6 & No & 2000 & 3 & 217 & 1750 \\
\hline 17 & 69 & $\mathrm{~F}$ & Kyphoscoliosis & 8 & T10-S1 & 7 & No & 1700 & 2 & 330 & 1687 \\
\hline 18 & 68 & F & Kyphoscoliosis & 8 & T10-S1 & 7 & No & 2500 & 3 & 344 & 3104 \\
\hline 19 & 71 & M & Kyphoscoliosis & 8 & T10-S1 & 6 & No & 3500 & 1 & 453 & 1764 \\
\hline
\end{tabular}

$\mathrm{mm}$ ) the Bone Scalpel has a long, narrow blade with a cutting surface measuring from $0.5 \mathrm{~mm}$ to $1.0 \mathrm{~mm}$ in thickness, and can make long, narrow precise cuts (8).

Sanborn et al. (8) studied the safety and efficacy of an ultrasonic bone-cutting device in an ovine model (12 sheep) compared to a control group (8 sheep in bone scalpel group and 4 in control group) using high-speed drill together with Kerrison punches and Leksell rongeurs. They showed a significant reduction in blood loss and operative time in the UBC group.

$\mathrm{Hu}$ et al. (2) reported that using ultrasonic bone scalpel in 128 consecutive spinal surgery patients decreases bone blood loss and operating time. The estimated blood loss was $425.4 \mathrm{~mL}$ for their patients undergoing posterior spinal procedures with an average of 5 levels being addressed. The average operative time was 4.3 hours with 2 complications directly related to the use of bone scalpel. The study was flawed, however, as they lacked a control group. Al-Mahfoudh et al. (10) similarly reported decreased blood loss and felt that the use of the bone scalpel had no effect on operating time. $74 \%$ had operations less than two hours with no mention of number of levels operated. They reported that the BS had a local hemostatic effect (sealing the bone surface) reducing bone bleeding. This study similarly missed a control group for analysis.

Gleizal et al. (9) used the ultrasonic bone scalpel in craniofacial surgical procedures including removal of the superior orbital roof in 30 cases of craniofaciostenosis, Le Fort III osteotomy for the treatment of Crouzon syndrome in two patients, and cutting the parietal and frontal bone in 30 cases of craniofaciostenosis. In this study they showed that the integrity of the adjacent soft tissue remained intact in all cases. The overall operative time however was increased (9). Hoigne et al. performed the first ultrasound osteotomy in hand surgery. In their study they concluded that the cuts were highly precise and there were no neurovascular complications associated with BS usage. The time needed for each cut was slightly (10 - 30 seconds) longer than the time needed with the use of a saw (11).

In this retrospective study, we found that both BS and control groups to be similar in terms of age, number of fusion levels, and ASA grade. In addition, there was no difference in rate of transfusion (14/19 of both study group and control group). Although the average blood transfused was $117 \mathrm{~mL}$ less in BS than the control group, this difference was not statistically significant. In this study, we also showed that both EBL and operative time of study group are less than the control group. However, none of these differences were statistically significant. There are several reasons for this, however. The bone scalpel patients were selected from a single center participating in the prospective adult spinal deformity study group while the control 

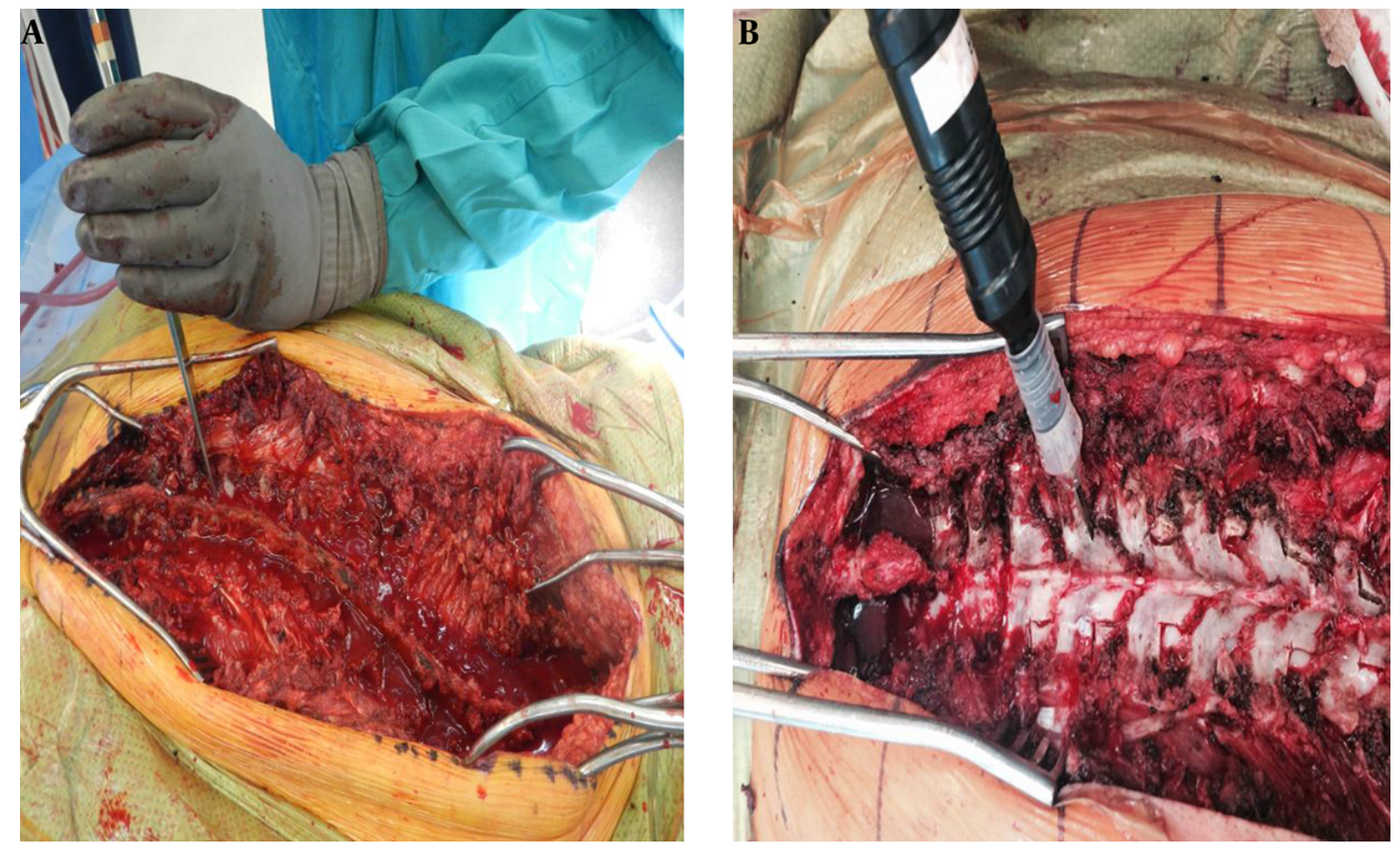

Figure 1. A, An osteotome is used to perform inferior articulating process facetectomies; B, the ultrasonic bone cutting device is used to perform inferior articulating process facetectomies.

group was selected from the group as a whole. The transfusion patterns and indications are very site dependent and could account for the lack of difference in transfusion rates. Although the blood loss was reduced by $43.1 \%$ or 703 $\mathrm{mL}$ in the BS group, this substantial decrease was not statistically significant, likely secondary to the small sample size. It is however, undeniable that a $703 \mathrm{~mL}$ reduction in blood loss is clinically relevant. The $43 \%$ blood loss reduction reported in our study is in line with 30\% - 40\% blood loss reduction reported by Bartley et al. (12).

In conclusion, this is the first report of the use of an ultrasonic bone-cutting device in adult spinal deformity. We have shown its use to be safe and effective with a $43 \%$ reduction in EBL without the addition of complications associated with the UBD. We did not identify a statistical difference in transfusion rates, EBL, or operative time. Despite the lack of statistical significance due to small sample size, the reduction of reported EBL by $43 \%$ makes a compelling argument to study the use of this device with a larger cohort to determine its role in ASD surgery.

\section{Footnotes}

Authors' Contribution: All authors were involved in study design, conducting, and supervising the study and statistical evaluations and manuscript preparation.

Funding/Support: Source of Funding: The Misonix BoneScalpel $^{\mathrm{TM}}$ was provided free of charge by Misonix, INC in this study.

\section{References}

1. Hara M, Takayasu M, Takagi T, Yoshida J. En bloc laminoplasty performed with threadwire saw. Neurosurgery. 2001;48(1):235-9. [PubMed: 11152356].

2. Hu X, Ohnmeiss DD, Lieberman IH. Use of an ultrasonic osteotome device in spine surgery: experience from the first 128 patients. Eur Spine J. 2013;22(12):2845-9. doi: 10.1007/s00586-013-2780-y. [PubMed: 23584231].

3. Hosono N, Miwa T, Mukai Y, Takenaka S, Makino T, Fuji T. Potential risk of thermal damage to cervical nerve roots by a high-speed drill.JBone Joint Surg Br. 2009;91(11):1541-4. doi: 10.1302/0301-620X.91B11.22196. [PubMed: 19880905].

4. Nakase H, Matsuda R, Shin Y, Park YS, Sakaki T. The use of ultrasonic bone curettes in spinal surgery. Acta Neurochir (Wien). 2006;148(2):207-12. doi: 10.1007/s00701-005-0655-7. [PubMed: 16311841]. 
5. Nakagawa H, Kim SD, Mizuno J, Ohara Y, Ito K. Technical advantages of an ultrasonic bone curette in spinal surgery. J Neurosurg Spine. 2005;2(4):431-5. doi:10.3171/spi.2005.2.4.0431. [PubMed: 15871482].

6. Flamm ES, Ransohoff J, Wuchinich D, Broadwin A. Preliminary experience with ultrasonic aspiration in neurosurgery. Neurosurgery. 1978;2(3):240-5. [PubMed: 732976].

7. Vercellotti T. Technological characteristics and clinical indications of piezoelectric bone surgery. Minerva Stomatol. 2004;53(5):207-14. [PubMed: 15263877].

8. Sanborn MR, Balzer J, Gerszten PC, Karausky P, Cheng BC, Welch WC. Safety and efficacy of a novel ultrasonic osteotome device in an ovine model. J Clin Neurosci. 2011;18(11):1528-33. doi: 10.1016/j.jocn.2011.04.016. [PubMed: 21917459].

9. Gleizal A, Bera JC, Lavandier B, Beziat JL. Piezoelectric osteotomy: a new technique for bone surgery-advantages in craniofacial surgery. Childs Nerv Syst. 2007;23(5):509-13. doi: 10.1007/s00381-006-0250-0. [PubMed: 17356890].

10. Al-Mahfoudh R, Qattan E, Ellenbogen JR, Wilby M, Barrett C, Pigott T. Applications of the ultrasonic bone cutter in spinal surgeryour preliminary experience. Br J Neurosurg. 2014;28(1):56-60. doi:
10.3109/02688697.2013.812182. [PubMed: 23841662].

11. Hoigne DJ, Stubinger S, Von Kaenel O, Shamdasani S, Hasenboehler P. Piezoelectric osteotomy in hand surgery: first experiences with a new technique. BMC Musculoskelet Disord. 2006;7:36. doi:10.1186/1471-24747-36. [PubMed:16611362].

12. Bartley CE, Bastrom TP, Newton PO. Blood loss reduction during surgical correction of adolescent idiopathic scoliosis utilizing an ultrasonic bone scalpel. Spine Deformity. 2014;2(4):285-90.

13. Satomi K, Ogawa J, Ishii Y, Hirabayashi K. Short-term complications and long-term results of expansive open-door laminoplasty for cervical stenotic myelopathy. Spine J. 2001;1(1):26-30. [PubMed: 14588365].

14. Sawamura Y, Fukushima T, Terasaka S, Sugai T. Development of a handpiece and probes for a microsurgical ultrasonic aspirator: instrumentation and application. Neurosurgery. 1999;45(5):1192-6. [PubMed: 10549937] discussion 1197.

15. Hadeishi H, Suzuki A, Yasui N, Satou Y. Anterior clinoidectomy and opening of the internal auditory canal using an ultrasonic bone curette. Neurosurgery. 2003;52(4):867-70. [PubMed: 12657183] discussion 870-1. 